\title{
A 2-EQUIVALENT KELLEY CONTINUUM
}

\author{
Carlos Islas \\ Universidad Autónoma de la Ciudad de México, Mexico
}

\begin{abstract}
The main purpose of this paper is to construct a 2equivalent compactification $X$ of a ray whose remainder is homeomorphic to $X$ and such that $X$ is a Kelley Continuum. In order to construct this example, we prove a theorem which gives conditions for an inverse limit of $\operatorname{arcs} X$ to be the compactification of a ray and $X$ is a Kelley continuum.
\end{abstract}

\section{INTRODUCTION}

We construct a 2-equivalent continuum which is a compactification $X$ of a ray whose remainder is homeomorphic to $X$ and such that $X$ is a Kelley continuum. In order to construct this example, we prove a theorem which gives conditions for an inverse limit of arcs to be the compactification of a ray and such that it is a Kelley continuum.

W. T. Ingram in [8, Theorem 2.3., p. 193] gives different conditions to obtain a Kelley continuum which is a compactification of a ray. R. A. Beane and W. J. Charatonik proved in [1, Theorem 2.3., p. 105] that for every chainable Kelley continuum $C$, there exists a compactification $D$ of a ray with remainder homeomorphic to $C$, and such that $D$ is a Kelley continuum.

A continuum is a compact and connected metric space, a map is a continuous function. Let $X$ and $Y$ be continua, a map $f: X \rightarrow Y$ is said to be confluent provided that for any subcontinuum $B$ of $Y$ and any component $A$ of $f^{-1}(B), f(A)=B$. A monotone map $f: X \rightarrow Y$, is a map such that $f^{-1}(C)$ is a connected set, for every connected subset $C$ of $Y$ (see [9, Lema 2.1 .12 , p. 74]). An arc means a space homeomorphic to the closed interval $[0,1]$. The set of positive integers is denoted by $\mathbb{N}$.

2010 Mathematics Subject Classification. 54F15, 54F50.

Key words and phrases. 2-equivalent continuum, Kelley Continuum, inverse limits.

The author thanks ICyTDF for the financial support provided. 
For a positive integer $n$, a continuum $X$ is said to be $n$-equivalent provided that $X$ contains exactly $n$ topologically distinct subcontinua. The arc and the pseudo-arc are the only known 1 -equivalent continua (see $[6,12,13])$. In [16], Whyburn has shown that each planar 1-equivalent continuum is tree-like, and the planarity assumption has been deleted after 40 years by Cook ([5]) who proved the tree-likeness of any 1-equivalent continuum. But it is still not known whether or not the arc and the pseudo-arc are the only 1-equivalent continua.

The class of 2-equivalent continua was studied by Mahavier in [11]. He proved that, if a 2-equivalent continuum contains an arc, then it is a simple triod, a simple closed curve or it is an irreducible continuum, and that the only locally connected 2-equivalent continua are a simple triod and a simple closed curve. In [11] it is also shown that if $X$ is a decomposable, not locally connected, 2-equivalent continuum containing an arc, then $X$ is arc-like and it is the compactification of a ray $R$ such that the remainder $K=\operatorname{cl}(R) \backslash R$ is a subcontinuum of $X$ (a ray is a space homeomorphic to the interval $[0, \infty)$ ). It is well known that the $\sin \frac{1}{x}$ continuum is an example of this kind of continua, such that the remainder $K$ is an arc, these are called Elsa continua. By a suggestion of the referee, we define that a continuum $X$ is n-equivalent compactification, if $X$ is a compactification of a ray and its remainder is n-equivalent. With this definition, we obtain that the $\sin \frac{1}{x}$ curve is a 1 equivalent compactification and in this paper we will present an example of a 2-equivalent compactification.

Let us recall some definitions and facts on inverse limits.

Let $\left\{X_{1}, X_{2}, X_{3}, \ldots\right\}$ be a sequence of continua and let $\left\{f_{1}^{2}, f_{2}^{3}, f_{3}^{4}, \ldots\right\}$ be a sequence of maps, such that $f_{i}^{i+1}: X_{i+1} \rightarrow X_{i}$ for every $i \in\{1,2, \ldots\}$. The sequence $\left\{X_{i}, f_{i}^{i+1}\right\}_{i=1}^{\infty}$ is called an inverse sequence and the inverse limit space is defined by

$X_{\infty}=\lim _{\longleftarrow}\left\{X_{n}, f_{n}^{n+1}\right\}=\left\{\left(x_{1}, x_{2}, \ldots\right)\right.$ : for every $\left.n \in \mathbb{N}, f_{n}^{n+1}\left(x_{n+1}\right)=x_{n}\right\}$,

as a subspace of the product $\prod_{n=1}^{\infty} X_{n}$.

Every space $X_{n}$ is called a factor space and $f_{n}^{n+1}$ a bonding map. We denote by $\pi_{i}: \lim \left\{X_{n}, f_{n}^{n+1}\right\} \rightarrow X_{i}$ the $i$-th projection map, restricted to the inverse limit. If $n>m$, with $n, m \in \mathbb{N}, f_{m}^{n}$ denotes the composition $f_{m}^{m+1} \circ \cdots \circ f_{n-1}^{n}$. Sometimes we use $f_{n}$ instead of $f_{n}^{n+1}$. If $K$ is a subcontinuum of $X_{\infty}$, we denote $K_{i}=\pi_{i}(K)$. If $X_{n}=X$, for every $n \in \mathbb{N}$, we denote $X_{\infty}=\lim _{\longleftarrow}\left\{X, f_{n}^{n+1}\right\}$ or $\lim _{\longleftarrow}\{X, f\}$ if every $f_{n}^{n+1}=f$. We will use the sequences $\left\{I_{1}, I_{2}, I_{3}, \ldots\right\}$ of subintervals of $I=[0,1]$, and sequences of maps $\left\{f_{1}^{2}, f_{2}^{3}, f_{3}^{4}, \ldots\right\}$, with $f_{i}^{i+1}\left(I_{i+1}\right)=I_{i}$. The Hilbert cube is a space homeomorphic to the product $\prod_{n=1}^{\infty} I_{n}$, where $I_{n}=[0,1]$ and the distance between two points $\left(x_{1}, x_{2}, x_{3}, \ldots\right),\left(y_{1}, y_{2}, y_{3}, \ldots\right)$ is defined by $d\left(\left(x_{1}, x_{2}, x_{3}, \ldots\right),\left(y_{1}, y_{2}, y_{3}, \ldots\right)\right)=\sum_{i=1}^{\infty} \frac{\left|x_{i}-y_{i}\right|}{2^{i}}$. 
Now, let us recall some facts about Kelley continua.

Definition 1.1. A continuum $X$ is a Kelley continuum at $p \in X$ if, for every $\varepsilon>0$ there exists $\delta>0$ such that, if $A$ is a subcontinuum of $X$, $p \in A, q \in X$ and $d(p, q)<\delta$, then there exists a subcontinuum $B$ of $X$ such that, $q \in B$ and $\mathcal{H}(A, B)<\varepsilon$ (d denotes the distance on $X$ and $\mathcal{H}$ denotes the Hausdorff distance on the hyperspace of subcontinua of $X, C(X)) . X$ is Kelley continuum if, $X$ is a Kelley continuum at every one of its points (see [3, p. 74]).

It is necessary to mention that a Kelley continuum is well known as a continuum with the property of Kelley.

THEOREM 1.2. [15, 16.11, p. 413] If $X$ is locally connected at $p$, then $X$ is a Kelley continuum at $p$.

Theorem 1.3. [4, Theorem 2, $p$. 190] If $X_{\infty}=\lim _{\longleftarrow}\left\{X_{n}, f_{n}^{n+1}\right\}$ and every factor space $X_{n}$ is a Kelley continuum and every bonding map $f_{n}^{n+1}$ is confluent, then $X_{\infty}$ is a Kelley continuum.

\section{TheOREM ON InVERse Limits}

2.1. A Theorem on Inverse Limits. The following theorem gives conditions under which an inverse limit of intervals is the compactification of a ray, another proof of Theorem 2.1 is in [2]. Nevertheless we include our proof for completeness and because the techniques are different.

TheOrem 2.1. Let $f: I \longrightarrow I$ be a map, where $I=[0,1]$, given by:

$$
f(x)= \begin{cases}4 x, & \text { if } \quad x \in\left[0, \frac{1}{4}\right] \\ \frac{3}{2}-2 x, & \text { if } \quad x \in\left[\frac{1}{4}, \frac{1}{2}\right]\end{cases}
$$

and $\left.\operatorname{Im} f\right|_{\left[\frac{1}{2}, 1\right]} \subseteq\left[\frac{1}{2}, 1\right]$ (Im denote the image). Let $X=\lim _{\longleftarrow}^{\lim }\{I, f\}$. Then $X$ is the compactification of a ray $R$ and $K=\lim _{\longleftarrow}\left\{\left[\frac{1}{2}, 1\right],\left.f\right|_{\left[\frac{1}{2}, 1\right]}\right\}$ is the remainder $\left(\left.f\right|_{\left[\frac{1}{2}, 1\right]}\right.$ means the restriction of the function $f$ on the set $\left.\left[\frac{1}{2}, 1\right]\right)$.

Proof. Let $X$ be as in the hypothesis. For every positive integer $n$, let

$$
\alpha_{n}=\left\{\left(x_{1}, x_{2}, \ldots\right) \in X: x_{n}<\frac{1}{2}\right\} .
$$

We note that:

1. $\alpha_{n} \subseteq \alpha_{n+1}$.

2. $\left.\pi_{n}\right|_{\alpha_{n}}$ is a homeomorphism from $\alpha_{n}$ onto $\left[0, \frac{1}{2}\right)$.

3. $x \in \alpha_{n} \backslash \alpha_{n-1}$ if and only if $x_{n} \in\left[\frac{1}{8}, \frac{1}{2}\right)$. 
We will show that $R=\cup_{n=1}^{\infty} \alpha_{n}$ is a ray. Observe that $R=\alpha_{1} \cup$ $\cup_{n=2}^{\infty}\left(\alpha_{n} \backslash \alpha_{n-1}\right)$, and define $\sigma: R \longrightarrow[0, \infty)$ by:

$$
\sigma(x)= \begin{cases}x_{1}, & \text { if } \quad x \in \alpha_{1} ; \\ x_{n}+\frac{3(n-1)}{8}, & \text { if } \quad x \in \alpha_{n} \backslash \alpha_{n-1}=\pi_{n}^{-1}\left[\frac{1}{8}, \frac{1}{2}\right) .\end{cases}
$$

We will prove that $\sigma$ is a homeomorphism. If $r \in[0, \infty)$, then either $r \in\left[0, \frac{1}{2}\right)=\sigma\left(\alpha_{1}\right)$ or there exists $n>1$ such that $r \in\left[\frac{3 n-2}{8}, \frac{3(n+1)-2}{8}\right)=$ $\sigma\left(\alpha_{n} \backslash \alpha_{n-1}\right)$. This proves that $\sigma$ is surjective.

We denote by $B_{n}$ the set $\sigma\left(\alpha_{n} \backslash \alpha_{n-1}\right)$, if $n>1$ and by $B_{1}=\left[0, \frac{1}{2}\right)=$ $\sigma\left(\alpha_{1}\right)$. We will see that $\sigma$ is injective. Since $B_{n} \cap B_{m}=\emptyset$ if $n \neq m$, the equality $\sigma(x)=\sigma(y)$ implies that either $x, y \in \alpha_{1}$ or $x, y \in \alpha_{n} \backslash \alpha_{n-1}$, $n>1$. Therefore, either $\sigma(x)=x_{1}=y_{1}=\sigma(y)$ in the first case or $\sigma(x)=$ $x_{n}+\frac{3(n-1)}{8}=y_{n}+\frac{3(n-1)}{8}=\sigma(y)$ in the second case. In both cases $x_{n}=y_{n}$ and since $\left.\pi_{n}\right|_{\alpha_{n}}$ is a homeomorphism, $x=y$.

To see that $\sigma$ is a continuous function we observe that the functions $\sigma_{\mid \alpha_{1}}$ and $\sigma_{\mid \alpha_{n} \backslash \alpha_{n-1}}$ are continuous. Then if either $x \in \pi_{1}^{-1}\left[0, \frac{1}{2}\right)=\alpha_{1}$ or $x \in \pi_{n}^{-1}\left(\frac{1}{8}, \frac{1}{2}\right)$, then $\sigma$ is continuous at $x$, since $\alpha_{1}$ and $\pi_{n}^{-1}\left(\frac{1}{8}, \frac{1}{2}\right)$ are open sets of $R$. It is only necessary to prove that $\sigma$ is continuous at every element of $\pi_{n}^{-1}\left(\frac{1}{8}\right)$. Let $x \in \pi_{n}^{-1}\left(\frac{1}{8}\right)$; i.e., $\pi_{n}(x)=x_{n}=\frac{1}{8}$. In this case

$$
\sigma(x)=x_{n}+\frac{3(n-1)}{8}=\frac{1}{8}+\frac{3(n-1)}{8}=\frac{3 n}{8}-\frac{1}{4} .
$$

Let $\varepsilon>0$ and choose positive numbers $\delta_{0}, \delta_{1}$ and $\delta_{2}$ with the following properties:

a) Since $\sigma_{\mid \alpha_{n} \backslash \alpha_{n-1}}$ is a map, if $y \in \alpha_{n} \backslash \alpha_{n-1}$ and $d(x, y)<\delta_{0}$, then $|\sigma(x)-\sigma(y)|<\varepsilon$.

b) If $s, t \in[0,1]$ and $|s-t|<\delta_{1}$, then $|f(s)-f(t)|<\varepsilon$.

c) If $y \in R$ and $d(x, y)<\delta_{2}$, then $\left|\pi_{n}(x)-\pi_{n}(y)\right|<\min \left\{\delta_{1}, \frac{3}{32}\right\}$.

Let $\delta=\min \left\{\delta_{0}, \delta_{2}\right\}$ and $y \in R$. We consider two cases:

CASE $1 \pi_{n}(y)=y_{n} \geq \frac{1}{8}$.

In this case $y \in \alpha_{n} \backslash \alpha_{n-1}$ and since $\delta \leq \delta_{0}$, it follows by a), that $|\sigma(x)-\sigma(y)|<\varepsilon$.

CASE $2 y_{n}<\frac{1}{8}$.

Since $d(x, y)<\delta_{2}$, by c), we have that $x_{n}-y_{n}<\frac{3}{32}, \frac{1}{8}>y_{n}>\frac{1}{32}$, $y_{n-1}>\frac{1}{8}$ and thus,

$$
\sigma(y)=y_{n-1}+\frac{3(n-2)}{8} .
$$

Now, since $x_{n}-y_{n}<\delta_{1}$, by b), we have that $\left|f\left(x_{n}\right)-f\left(y_{n}\right)\right|<\varepsilon$. It is sufficient to show that $|\sigma(x)-\sigma(y)|=\left|f\left(x_{n}\right)-f\left(y_{n}\right)\right|$. Since $f\left(x_{n}\right)=\frac{1}{2}$ and $f\left(y_{n}\right)=y_{n-1}<\frac{1}{8}<\frac{1}{2}$, then $\left|f\left(x_{n}\right)-f\left(y_{n}\right)\right|=\frac{1}{2}-y_{n-1}$.

By (1) and (2), $\sigma(x)-\sigma(y)=\frac{1}{2}-y_{n-1}$. Therefore, $\sigma$ is continuous. 
To show that the function $\sigma^{-1}:[0, \infty) \rightarrow R$ is continuous, we define $\lambda:[0, \infty) \rightarrow\left[0, \frac{1}{2}\right)$ by

$$
\lambda(t)= \begin{cases}t, & \text { if } t \in B_{1} \\ t-\frac{3(n-1)}{8}, & \text { if } t \in B_{n} \text { and } n>1 .\end{cases}
$$

Recall that $B_{1}=\left[0, \frac{1}{2}\right)=\sigma\left(\alpha_{1}\right)$ and $B_{n}=\left[\frac{3 n-2}{8}, \frac{3(n+1)-2}{8}\right)=\sigma\left(\alpha_{n} \backslash \alpha_{n-1}\right)$.

It is easy to verify the following:

1) $\left.\lambda\right|_{B_{n}}: B_{n} \rightarrow\left[0, \frac{1}{2}\right)$ is continuous, for every $n$.

2) If $n>1$ and $t \in B_{n}$, then $\frac{1}{8} \leq \lambda(t)<\frac{1}{2}$.

3) If $t \in B_{n}$, then $\pi_{n}^{-1}(\lambda(t))$ has exactly one point in $\bigcup_{m=1}^{\infty} \alpha_{m}$ and $\sigma^{-1}(t)=\pi_{n}^{-1}(\lambda(t)) \in \alpha_{n} \backslash \alpha_{n-1} \subset R$.

By 3$),\left.\sigma^{-1}\right|_{B_{n}}$ is continuous for every $n$. Since $[0, \infty)=\cup_{n=1}^{\infty} B_{n}$, we only have to prove that $\sigma^{-1}$ is continuous at the points of the form $\frac{3 n-2}{8}$. Given $x \in[0, \infty)$ we denote $x_{n}=\pi_{n}\left(\sigma^{-1}(x)\right)$. Let $t=\frac{3 n-2}{8}$. Then $\frac{1}{8}=\lambda(t)$, let $t_{n}=\lambda(t), t_{1}=t_{2}=t_{3}=\cdots=t_{n-1}=\frac{1}{2}$ and $t_{n+j}=f^{-j}\left(\frac{1}{8}\right)=\frac{1}{4 j}\left(\frac{1}{8}\right)$ if $j \geq 1$. Let $\varepsilon>0$ and $\delta^{\prime}>0$ such that, if $\left|u-\frac{1}{2}\right|<\delta^{\prime}$ then $\left|f^{k}(u)-f^{k}\left(\frac{1}{2}\right)\right|<\varepsilon$ for every $k \in\{1,2, \ldots, n-2\}$. Let $\delta=\min \left\{\frac{1}{8}, \varepsilon, \delta^{\prime}\right\}$. If $s \in[0, \infty)$ and $|s-t|<\delta$, then, since $\delta \leq \frac{1}{8}, s \in B_{n-1} \cup B_{n}$. Since $\left.\sigma^{-1}\right|_{B_{n}}$ is continuous, we only have to consider the case when $s \in B_{n-1}$. We will prove that $\left|s_{j}-t_{j}\right|<\varepsilon$ for every $j \in \mathbb{N}$. Since $s_{n-1}=\lambda(s)=s-\frac{3(n-1)-2}{8}$, $\left|s_{n-1}-t_{n-1}\right|=\left|\left(s-\frac{3(n-1)-2}{8}\right)-\frac{1}{2}\right|=\left|s-\frac{(3 n-2)}{8}\right|=|s-t|<\delta \leq \varepsilon$. Now, since $t_{n-1}=\frac{1}{2}$, the choice of $\delta^{\prime}$ implies that $\left|f^{k}\left(s_{n-1}\right)-f^{k}\left(\frac{1}{2}\right)\right|=$ $\left|s_{n-1-k}-t_{n-1-k}\right|<\varepsilon$, for every $k \in\{1,2, \ldots, n-2\}$; i.e., $\left|s_{j}-t_{j}\right|<\varepsilon$ if $j \in\{1,2, \ldots, n-2\}$. Since $s_{n-1}, t_{n-1} \in\left[0, \frac{1}{2}\right)$ and $f^{-1}(u)=\frac{u}{4}$ if $u \in\left[0, \frac{1}{2}\right)$, we have that $\left|f^{-k}\left(s_{n-1}\right)-f^{-k}\left(t_{n-1}\right)\right|=\left|\frac{s_{n-1}-t_{n-1}}{4^{k}}\right|<\frac{\varepsilon}{4^{k}}<\varepsilon$, for every $k>n-1$. Thus, $\left|s_{j}-t_{j}\right|<\varepsilon$ if $j \geq n$. Then $\sigma^{-1}$ is continuous.

Let us note that $f\left(\left[\frac{1}{2}, 1\right]\right) \subset\left[\frac{1}{2}, 1\right]$. Then we define:

$$
K=\lim _{\longleftarrow}\left\{\left[\frac{1}{2}, 1\right],\left.f\right|_{\left[\frac{1}{2}, 1\right]}\right\} .
$$

Then $X=R \cup K$ and $R \cap K=\emptyset$.

We will show that $\bar{R} \backslash R=K$. We only have to prove that $K \subseteq \bar{R}$. By the definition of the distance, if $u, v \in X=\lim \{I, f\}$ and, for some $k \in \mathbb{N}$, $u_{k}=v_{k}$, then $d(u, v)<\frac{1}{2^{k}}$.

Let $x \in K$. Then $x_{n} \in\left[\frac{1}{2}, 1\right]$ for every $n \in \mathbb{N}$. Let $\varepsilon>0$ and $n \in \mathbb{N}$ such that $\frac{1}{2^{n}}<\varepsilon$. By definition of $f, f\left(\left[0, \frac{1}{2}\right)\right)=[0,1]$. Then there exists $y \in\left[0, \frac{1}{2}\right)$ such that $f(y)=x_{n}$. Hence, there is a point $p_{n}$ in $\alpha_{n+1}$ such that, its $(n+1)$-th coordinate is $y$, which implies that $d\left(x, p_{n}\right)<\frac{1}{2^{n}}<\varepsilon$. Thus, $K \subset \bar{R}$. Therefore, $X$ is a compactification of a ray $R$ such that $\bar{R} \backslash R=K$, which is a continuum. 
2.2. Main Theorem on Inverse Limits. To prove the main Theorem we need the following Lemma.

Lemma 2.2. [7, Lemma 2.2, p. 193] Let $M=\lim _{\longleftarrow}\left\{X_{i}, f_{i}\right\}$. Assume that, for every $i \in \mathbb{N}, X_{i}$ is a continuum and $\varepsilon>0$. Then there exist a positive integer $N$ and a positive number $\delta$, such that, if $H$ and $K$ are subcontinua of $M$, such that $\mathcal{H}_{N}\left(H_{N}, K_{N}\right)<\delta$, then $\mathcal{H}(H, K)<\varepsilon\left(\mathcal{H}_{N}\right.$ and $\mathcal{H}$ denote the Hausdorff distance on $C\left(X_{N}\right)$ and $C(M)$, respectively).

Theorem 2.3. Let $X=\lim _{\longleftarrow}\{I, f\}$, where $I=[0,1]$ and $f: I \rightarrow I$ is a map such that:

1. $f(x)= \begin{cases}4 x, & \text { if } x \in\left[0, \frac{1}{4}\right] ; \\ \frac{3}{2}-2 x, & \text { if } x \in\left[\frac{1}{4}, \frac{1}{2}\right] .\end{cases}$

2. $\left.\operatorname{Im} f\right|_{\left[\frac{1}{2}, 1\right]}=\left[\frac{1}{2}, 1\right]$;

3. $C=\lim _{\longleftarrow}\left\{\left[\frac{1}{2}, 1\right],\left.f\right|_{\left[\frac{1}{2}, 1\right]}\right\}$ is a Kelley continuum.

Then $X$ is a compactification of a ray with remainder $C$ and $X$ is a Kelley continuum.

Proof. By Theorem 2.1, $X$ is a compactification of a ray and its remainder is $C$.

i) Note that, if $p$ is a point of the ray, then, by Theorem $1.2, X$ is a Kelley continuum at $p$.

Let $\varepsilon>0$.

ii) Since $C$ is a Kelley continuum, there exists a positive number $\delta_{1}$ such that, if $A$ is a subcontinuum of $C, r$ is a point of $A$ and $s$ is a point of $C$ and $d(r, s)<\delta_{1}$, then there exists a subcontinuum $B$ of $C$ with $s$ in $B$ and $\mathcal{H}(A, B)<\frac{\varepsilon}{3}$.

Let $\varepsilon_{1}$ be a positive number, such that, $\varepsilon_{1}<\min \left\{\frac{\varepsilon}{3}, \delta\right\}$.

iii) By Lemma 2.2, there exist a positive number $\delta_{2}<\delta_{1}$ and a positive integer $N$ such that, if $H$ and $K$ are subcontinua of $X$ with $\mathcal{H}_{N}\left(H_{N}, K_{N}\right)<$ $\delta_{2}$, we have that $\mathcal{H}(H, K)<\varepsilon_{1}$.

iv) Since $f$ is uniformly continuous, there exists a positive number $\delta_{3}$ such that if $x$ and $y$ are points of $X_{N+1}$ with $|x-y|<\delta_{3}$, then $|f(x)-f(y)|<\frac{\delta_{2}}{2}$.

Let $\delta=\frac{\delta_{3}}{2^{N+1}}$ and suppose that $H$ is a subcontinuum of $X$. Let $p \in H$ and $q \in X$ such that $d(p, q)<\delta$. Therefore, $d(p, q)<\frac{\delta_{3}}{2^{N+1}}$ and $\left|p_{N+1}-q_{N+1}\right|<$ $\delta_{3}$.

Let $J$ be the arc irreducible respect to $H_{N+1} \cup\left\{q_{N+1}\right\}$; i.e., $J$ is the arc such that $H_{N+1} \cup\left\{q_{N+1}\right\} \subset J$ and, if $A$ is a subarc such that $H_{N+1} \cup\left\{q_{N+1}\right\} \subset$ $A$, then $J \subset A$.

We will see that $\mathcal{H}_{N}\left(H_{N}, f(J)\right) \leq \frac{\delta_{2}}{2}<\delta_{2}$. Let $x \in f(J)$, then there exists $y \in J$ such that $x=f(y)$. Now, since $y \in J$, there exists $z \in H_{N+1}$ such that $|y-z|<\left|p_{N+1}-q_{N+1}\right|$ (by the irreducibility of $J$ ). Then $|y-z|<\delta_{3}$ and $|f(y)-f(z)|<\frac{\delta_{2}}{2}<\delta_{2}$. In consequence, for every element $x$ in $f(J)$, 
there exists $f(z)$ in $H_{N}$ such that $|x-f(z)|<\frac{\delta_{2}}{2}$, and, since $H_{N} \subset f(J)$, $\mathcal{H}_{N}\left(H_{N}, f(J)\right)<\delta_{2}$.

We will consider two cases:

CASE 1. $q_{N+2}<\frac{1}{2}$.

In this case we have two possibilities

CASE 1.1. $H_{N+1}$ contains 1 .

By the definition of $f$, there exists a subinterval of $I,[x, y] \subset\left[0, \frac{1}{2}\right]$ such that $f([x, y])=J$, moreover, we could choose $[x, y]$ in such a way that $q_{N+2} \in[x, y]$, because $q_{N+1} \in J$ and $q_{N+2}<\frac{1}{2}$. Now, define $K$ as the subcontinuum of $X$ such that $K_{N+2}=[x, y]$ and

$$
K_{i}= \begin{cases}f^{N+2-i}([x, y]), & \text { if } \quad i<N+2 \\ f^{-(i-(N+2))}([x, y]), & \text { if } i>N+2 .\end{cases}
$$

Thus, $f\left(K_{i+1}\right)=K_{i}$ and $K \subset X$. Moreover, $q \in K, K_{N}=f(J)$ and, since $\mathcal{H}\left(H_{N}, f(J)\right)<\delta_{2}, \mathcal{H}(H, K)<\frac{\varepsilon}{3}$.

CASE $1.21 \notin H_{N+1}$.

Consider two possibilities.

a) $H_{N+1} \subset\left[\frac{1}{2}, 1\right]$, in this case, we choose: $[x, y] \subset\left[0, \frac{1}{4}\right]$ such that $f([x, y])=J$ if $q_{N+2} \leq \frac{1}{4}$ or $[x, y] \subset\left[\frac{1}{4}, \frac{1}{2}\right]$ such that $f([x, y])=J$ if $\frac{1}{4}<$ $q_{N+2}<\frac{1}{2}$. Now, define $K$ in the same way as in Case 1.1. We obtain that $\mathcal{H}(H, K)<\varepsilon$.

b) If $H_{N+1} \cap\left[0, \frac{1}{2}\right) \neq \emptyset$, since $H_{N+2}$ is a continuum and $H_{N+2} \cap\left[0, \frac{1}{4}\right) \neq \emptyset$, $H_{N+2} \subset\left[0, \frac{1}{4}\right)$, so $H \subset \alpha_{N+2}$, with $\alpha_{N+2}=\left\{\left(x_{1}, \ldots\right) \in X \mid x_{N+2}<\frac{1}{2}\right\}$ $\left(\alpha_{N+2}\right.$ is the subset of the ray defined in Theorem 2.1). In this case we obtain that $p \in H \subset \alpha_{N+2}$; hence, $p$ is an element of the ray and, by i), $X$ has the property of Kelley in $p$.

CASE $2 q_{N+2} \geq \frac{1}{2}$.

In this case, we have two possibilities:

CASE $2.1 J \subseteq\left[\frac{1}{2}, 1\right]$.

Since $q_{N+2} \geq \frac{1}{2}$ and $J \subseteq\left[\frac{1}{2}, 1\right], H_{N+1} \subseteq\left[\frac{1}{2}, 1\right]$. We define $A$ as follows: If $H_{i} \subseteq\left[\frac{1}{2}, 1\right]$ for every $i \in \mathbb{N}$, we define $A=H$. If $H_{i} \cap\left[0, \frac{1}{2}\right) \neq \emptyset$ for some $i$, we define $A$ in the following way:

Let $j+1=\min \left\{i \in \mathbb{N}: H_{i} \cap\left[0, \frac{1}{2}\right) \neq \emptyset\right\}$ (it is clear that $j>N+1$ ). By $\left[14,13.71\right.$, p. 310], $\left.f\right|_{\left[\frac{1}{2}, 1\right]}$ is weakly confluent. Then we may choose a

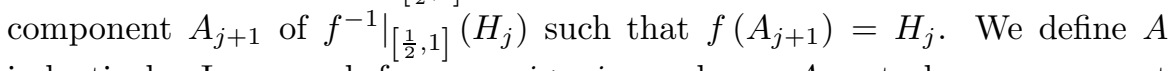
inductively. In general, for every $i>j$, we choose $A_{i+1}$ to be a component of $\left.f^{-1}\right|_{\left[\frac{1}{2}, 1\right]}\left(A_{i}\right)$ such that $f\left(A_{i+1}\right)=A_{i}$. Hence, $A=\lim _{\longleftarrow}\left\{A_{i},\left.f\right|_{A_{i+1}}\right\}$ is a subcontinuum of $C$, which coincides with $H$ at least in the first $N+1$ coordinates. By the choice of $\delta$, by iii) and ii), we have that $\mathcal{H}(A, H)<\frac{\varepsilon}{3}$.

We define $r$ and $s$ in the same way: $r$ and $s$ are elements of $C$, which coincide with $p$ and $q$ respectively in at least the first $N+1$ coordinates. In 
fact, $s$ coincides with $q$ at least in the first $N+2$ coordinates, because of our assumption; i.e., $J \subseteq\left[\frac{1}{2}, 1\right]$.

Since $\left|r_{N+1}-s_{N+1}\right|<\delta_{3}$ we have $\left|r_{N}-s_{N}\right|<\delta_{2}$. By the choice of $\delta_{2}$, $d(r, s)<\delta_{1}$ and since $C$ is a Kelley continuum, there exists a subcontinuum $B$ of $C$ such that $s \in B$ and $\mathcal{H}(A, B)<\frac{\varepsilon}{3}$. Thus, $\mathcal{H}(H, B)<\frac{2 \varepsilon}{3}$. If $q \in B$, we choose $K=B$ to obtain the conclusion. Moreover, since $C$ is a Kelley continuum, if $q \in C$, we also obtain the conclusion. Suppose that $q \notin B$ and that there exists $i>N+2$ such that $q_{i}<\frac{1}{2}$.

Let $j+1=\min \left\{i \in \mathbb{N}: q_{i}<\frac{1}{2}\right\}$. We will construct $K$ as in Case 1.1.

Since $f\left(\left[0, \frac{1}{2}\right]\right)=[0,1]$, there is a subinterval $[x, y]$ contained either in $\left[\frac{1}{8}, \frac{1}{4}\right]$ or in $\left[\frac{1}{4}, \frac{1}{2}\right]$ such that $q_{j+1} \in[x, y]$ and such that $f([x, y])=B_{j}$.

Let $K_{j+1}=[x, y], K_{j+2}=f^{-1}\left(K_{j+1}\right), K_{j+i+1}=f^{-1}\left(K_{j+i}\right)$, for every

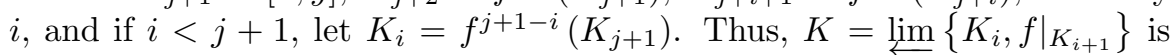
a continuum containing $q$ and such that $K_{N}=B_{N}$. Since $K_{j}=B_{j}$, by iii), $\mathcal{H}(H, K)<\varepsilon$.

CASE $2.2 J \cap\left[0, \frac{1}{2}\right) \neq \emptyset$.

We have $q_{N+2} \geq \frac{1}{2}$ and $J \cap\left[0, \frac{1}{2}\right) \neq \emptyset$.

If $f(J) \subseteq\left[\frac{1}{2}, 1\right]$, then we proceed as in case 2.1, using $f(J)$ instead of $J$.

If $f(J) \cap\left[0, \frac{1}{2}\right) \neq \emptyset$, there exists an element $y<\frac{1}{2}$ in $f(J)$, and by the definition of $f, y=f(x)$, for some $x \in J$, where $x<\frac{1}{4}$, now, since $q_{N+2} \geq \frac{1}{2}$, $q_{N+1} \geq \frac{1}{2}$, so $f\left(\left[\frac{1}{4}, \frac{1}{2}\right]\right) \subseteq J$, thus $\left[\frac{1}{2}, 1\right] \subseteq f(J)$.

Let $y_{0}=\min \{y: y \in f(J)\}$. Then $f(J)=\left[y_{0}, 1\right]$. Let $K_{N}=f(J)$, $K_{N+1}=f^{-1}\left(K_{N}\right)=\left[\frac{y_{0}}{4}, 1\right], K_{N+2}=f^{-1}\left(K_{N+1}\right)=\left[\frac{y_{0}}{16}, 1\right], \ldots$ If $i<$ $N, K_{i}=f^{N-i}\left(K_{N}\right)$. Let $K=\lim \left\{K_{i},\left.f\right|_{K_{i+1}}\right\}$. Then $K$ is a continuum containing $q$.

Since $q \in K$ and $\mathcal{H}\left(H_{N}, f(J)\right)<\delta_{2}$ and $K_{N}=f(J)$, we obtain by iii), that $\mathcal{H}(H, K)<\varepsilon$.

\section{A 2-equivalent Kelley continuum.}

In this section, we describe the factor spaces of our example, which is a 2equivalent continuum with the following properties: It is the compactification of a ray and its remainder is homeomorphic to the whole space and it is a Kelley continuum. Then we give some properties of the factor spaces and the bonding maps. We show the continuity and the confluence of the bonding maps. Finally, we construct the example and prove its properties.

3.1. Factor spaces. The function $g$, that we define below, will help us to define the factor spaces:

Let $g:\left[\frac{1}{2}, 1\right] \longrightarrow[0,1]$ defined by $g(x)=2 x-1$ and let $g^{-1}$ its inverse. 
Let $X_{1}=\lim _{\longleftarrow}\left\{I, f_{1}\right\}$, where $I=[0,1]$ and $f_{1}: I \longrightarrow I$ is defined by:

$$
f_{1}(x)= \begin{cases}4 x, & \text { if } \quad x \in\left[0, \frac{1}{4}\right] ; \\ \frac{3}{2}-2 x, & \text { if } x \in\left[\frac{1}{4}, \frac{1}{2}\right] ; \\ \frac{1}{2}, & \text { if } x \in\left[\frac{1}{2}, 1\right] .\end{cases}
$$

Then, by Theorem 2.1, $X_{1}$ is homeomorphic to the interval [0,1], since $X_{1}$ is the compactification of a ray $R_{1}$ with remainder $K_{1}=\lim _{\longleftarrow}\left\{\left[\frac{1}{2}, 1\right],\left.f_{1}\right|_{\left[\frac{1}{2}, 1\right]}\right\}=$ $\left\{\left(\frac{1}{2}, \frac{1}{2}, \ldots\right)\right\}$. Recall that

$$
X_{1}=\left\{\left(\frac{1}{2}, \frac{1}{2}, \ldots\right)\right\} \cup\left(\cup_{n=1}^{\infty} \alpha_{n}^{1}\right) \text { where } \alpha_{n}^{1}=\left\{x \in X_{1}: x_{n}<\frac{1}{2}\right\} .
$$

It is easy to see that $X_{1}$ is a Kelley continuum.

Let $X_{2}=\lim _{\longleftarrow}\left\{I, f_{2}\right\}$, with $f_{2}: I \longrightarrow I$ defined by

$$
f_{2}(x)= \begin{cases}4 x, & \text { if } x \in\left[0, \frac{1}{4}\right] ; \\ \frac{3}{2}-2 x, & \text { if } x \in\left[\frac{1}{4}, \frac{1}{2}\right] ; \\ g^{-1}\left(f_{1}(g(x))\right), & \text { if } x \in\left[\frac{1}{2}, 1\right] .\end{cases}
$$

By Theorem 2.1, $X_{2}=K_{2} \cup R_{2}$ where $K_{2}=\varliminf_{\longleftarrow}\left\{\left[\frac{1}{2}, 1\right],\left.f_{2}\right|_{\left[\frac{1}{2}, 1\right]}\right\}$ and $R_{2}=$ $\sum_{n=1}^{\infty} \alpha_{n}^{2}$ where $\alpha_{n}^{2}=\left\{x \in X_{2}: x_{n}<\frac{1}{2}\right\}$; i.e., $X_{2}$ is the compactification of a ray with remainder $K_{2}$. Note that $K_{2}$ is homeomorphic to $X_{1}$; hence, $X_{2}$ satisfies the hypotheses of the Theorem 2.3, then $X_{2}$ is a Kelley continuum.

In general, we define, for every positive integer $n, X_{n+1}=\varliminf_{\longleftarrow}\left\{I, f_{n+1}\right\}$, where $I=[0,1]$ and $f_{n+1}: I \longrightarrow I$, given by

$$
f_{n+1}(x)= \begin{cases}4 x, & \text { if } x \in\left[0, \frac{1}{4}\right] ; \\ \frac{3}{2}-2 x, & \text { if } x \in\left[\frac{1}{4}, \frac{1}{2}\right] ; \\ g^{-1}\left(f_{n}(g(x))\right), & \text { if } x \in\left[\frac{1}{2}, 1\right] .\end{cases}
$$

Then $X_{n+1}$ is a compactification of a ray $R_{n+1}$ such that its remainder, $K_{n+1}=\lim _{\longleftarrow}\left\{\left[\frac{1}{2}, 1\right],\left.f_{n+1}\right|_{\left[\frac{1}{2}, 1\right]}\right\}$ is homeomorphic to $X_{n}$ and, by Theorem $2.3, X_{n+1}$ is a Kelley continuum.

3.2. Properties of the factor spaces. We analyze the space $X_{i}$. As in the proof of Theorem 2.1, let

$$
\alpha_{n}^{i}=\left\{\left(x_{1}, x_{2}, \ldots\right) \in X_{i}: x_{n}<\frac{1}{2}\right\} .
$$

Then:

1. $\alpha_{n}^{i} \subset \alpha_{n+1}^{i}$,

2. $\left.\pi_{n}\right|_{\alpha_{n}^{i}}$ is a homeomorphism from $\alpha_{n}^{i}$ onto $\left[0, \frac{1}{2}\right)$, 
3. $x \in \alpha_{n}^{i} \backslash \alpha_{n-1}^{i}$ if and only if $x_{n} \in\left[\frac{1}{8}, \frac{1}{2}\right)$,

4. $R_{i}=\cup_{n=1}^{\infty} \alpha_{n}^{i}$ is a ray and $R_{i}=\alpha_{1}^{i} \cup \cup_{n=2}^{\infty}\left(\alpha_{n}^{i} \backslash \alpha_{n-1}^{i}\right)$, and

5. $X_{i}=R_{i} \cup K_{i}$, where $K_{i}=\overline{R_{i}} \backslash R_{i}, \overline{R_{i}}=X_{i}$ and $K_{i}=\lim _{\longleftarrow}\left\{\left[\frac{1}{2}, 1\right],\left.f_{i}\right|_{\left[\frac{1}{2}, 1\right]}\right\}$.

If $i>1$, observe that $K_{i}$ is homeomorphic to $X_{i-1}$ with the homeomorphism $\left.\bar{g}\right|_{K_{i}}$, where $\bar{g}:\left[\frac{1}{2}, 1\right]^{\infty} \longrightarrow I^{\infty}$ is defined by $\bar{g}\left(\left(x_{1}, x_{2}, \ldots\right)\right)=$ $\left(g\left(x_{1}\right), g\left(x_{2}\right), \ldots\right)$. Let us note that the function $\bar{g}:\left[\frac{1}{2}, 1\right]^{\infty} \rightarrow I^{\infty}$ is continuous, since it is continuous at each coordinate. Moreover, in the same way, we may define the map $\bar{g}^{-1}$ and thus, this map is continuous too.

We observe that $\alpha_{1}^{i}=\pi_{1}^{-1}\left(\left[0, \frac{1}{2}\right)\right)$ and $\alpha_{n}^{i} \backslash \alpha_{n-1}^{i}=\pi_{n}^{-1}\left(\left[\frac{1}{8}, \frac{1}{2}\right)\right)$.

3.3. The bonding maps.

3.3.1. The map $g_{1}$. We define the function $g_{1}: X_{2} \rightarrow X_{1}$ by:

$g_{1}(x)= \begin{cases}x, & \text { if } x \in \alpha_{2}^{2} \\ \left(y_{1}, y_{2}, \ldots, y_{n-2}, x_{n-1}, x_{n}, x_{n+1}, \ldots\right), & \text { if } x \in \alpha_{n}^{2} \backslash \alpha_{n-1}^{2}, n>2 \\ \left(\frac{1}{2}, \frac{1}{2}, \ldots\right), & \text { if } x \in K_{2}\end{cases}$

where $y_{i}=f_{1}^{n-1-i}\left(x_{n-1}\right)$, and $x=\left(x_{1}, x_{2}, x_{3}, \ldots\right) \in X_{2}$. We observe that $g_{1}$ is well defined, because $\left.f_{1}\right|_{\left[0, \frac{1}{2}\right]}=\left.f_{2}\right|_{\left[0, \frac{1}{2}\right]}$.

3.3.2. Continuity of $g_{1}$ on the ray. We will see that $g_{1}$ is continuous.

We will prove the continuity of $g_{1}$ on the ray $R_{2}$. Since $\left.g_{1}\right|_{\alpha_{2}^{2}}$ is the identity, $g_{1}$ is continuous at $x \in \alpha_{2}^{2}$, because $x \in \pi_{2}^{-1}\left(\left[0, \frac{1}{2}\right)\right)$, which is an open set of $X_{2}$. In the same way, $\left.g_{1}\right|_{\pi_{n}^{-1}\left(\frac{1}{8}, \frac{1}{2}\right)}$ is continuous, since it is continuous at each coordinate and the set $\pi_{n}^{-1}\left(\frac{1}{8}, \frac{1}{2}\right)$ is open in $X_{2}$. Therefore, if $x \in \pi_{n}^{-1}\left(\frac{1}{8}, \frac{1}{2}\right)$, $g_{1}$ is continuous at $x$. Now, we only have to prove the continuity of $g_{1}$ at every $x \in \pi_{n}^{-1}\left(\frac{1}{8}\right) \subset R^{2}$.

Let $x=\left(x_{1}, x_{2}, \ldots\right) \in \pi_{n}^{-1}\left(\frac{1}{8}\right)$; i.e., $\pi_{n}(x)=x_{n}=\frac{1}{8}$. By the definition of $X_{2}, x_{n-1}=f_{2}\left(x_{n}\right)=4\left(\frac{1}{8}\right)=\frac{1}{2}$. Then $x \in \alpha_{n}^{2} \backslash \alpha_{n-1}^{2}$ and thus $g_{1}(x)=$ $\left(\frac{1}{2}, \ldots, \frac{1}{2}, \frac{1}{2}, \frac{1}{8}, \ldots\right)$; i.e., $g_{1}(x)=x$.

Let $\varepsilon>0$. Then there exist positive numbers $\delta_{0}, \delta_{1}$ and $\delta_{2}$ less than $\varepsilon$ such that:

a) Since $\left.g_{1}\right|_{\alpha_{n}^{2} \backslash \alpha_{n-1}^{2}}$ is continuous, if $y \in \alpha_{n}^{2} \backslash \alpha_{n-1}^{2}$ and $d(x, y)<\delta_{0}$, then $d\left(g_{1}(x), g_{1}(y)\right)<\varepsilon$.

b) By the uniform continuity of $f_{1}$. If $s, t \in[0,1]$ and $|s-t|<\delta_{1}$, then $\sum_{i=1}^{n-2} \frac{\left|f_{1}^{n-i}(s)-f_{1}^{n-i}(t)\right|}{2^{i-1}}<\frac{\varepsilon}{4}$.

c) If $y \in R_{2}$ and $d(x, y)<\delta_{2}$, then $\left|\pi_{n}(x)-\pi_{n}(y)\right|<\min \left\{\delta_{1}, \frac{1}{16}\right\}$.

Let $\delta=\min \left\{\delta_{0}, \delta_{2}\right\}$ and $y \in X_{2}$ such that $d(x, y)<\delta$. We consider two cases:

Case $1 \pi_{n}(y)=y_{n} \geq \frac{1}{8}$. 
In this case $y \in \alpha_{n}^{2} \backslash \alpha_{n-1}^{2}$ and since $\delta \leq \delta_{0}$, by a), $d\left(g_{1}(x), g_{1}(y)\right)<\varepsilon$. Case $2 \pi_{n}(y)=y_{n}<\frac{1}{8}$.

Since $\left.d(x, y)<\delta_{2}, \mathrm{c}\right)$ implies that $x_{n}-y_{n}<\frac{1}{16}$; i.e., either $\frac{1}{8}>$ $y_{n}>x_{n}-\frac{1}{16}$ or $\frac{1}{8}>y_{n}>\frac{1}{16}$. Then $\frac{1}{2}>y_{n-1}>\frac{4}{16}=\frac{1}{4}>\frac{1}{8}$, since $y_{n-1}=f_{2}\left(y_{n}\right)$ and $f_{2}(x)=4 x$ if $x \in\left[0, \frac{1}{4}\right]$. This implies that $y \in$ $\alpha_{n-1}^{2} \backslash \alpha_{n-2}^{2}$, hence, $g_{1}(y)=\left(f_{1}^{n-3}\left(y_{n-2}\right), \ldots, f_{1}\left(y_{n-2}\right), y_{n-2}, y_{n-1}, \ldots\right)=$ $\left(f_{1}^{n-1}\left(y_{n}\right), \ldots, f_{1}\left(y_{n}\right), y_{n}, \ldots\right)$.

Now, since $f_{1}=f_{2}$ on $\left[0, \frac{1}{2}\right)$,

$$
\begin{aligned}
d\left(g_{1}(x), g_{1}(y)\right)=d( & \left(f_{1}^{n-1}\left(x_{n}\right), \ldots, f_{1}\left(x_{n}\right), \frac{1}{8}, \ldots\right) \\
& \left.\left(f_{1}^{n-3}\left(y_{n-2}\right), \ldots, f_{1}\left(y_{n-2}\right), y_{n-2}, y_{n-1}, \ldots\right)\right)
\end{aligned}
$$

Since $y_{n-2}=f_{1}^{2}\left(y_{n}\right)$, we have that

$$
\begin{aligned}
d\left(g_{1}(x), g_{1}(y)\right) & =\sum_{i=1}^{n-1} \frac{\left|f_{1}^{n-i}\left(x_{n}\right)-f_{1}^{n-i}\left(y_{n}\right)\right|}{2^{i}}+\sum_{i=1}^{\infty} \frac{\left|x_{i}-y_{i}\right|}{2^{i}} \\
& =\sum_{i=1}^{n-1} \frac{\left|f_{2}^{n-i}\left(x_{n}\right)-f_{2}^{n-i}\left(y_{n}\right)\right|}{2^{i}}+\sum_{i=n}^{\infty} \frac{\left|x_{i}-y_{i}\right|}{2^{i}} \\
& <\frac{\varepsilon}{4}+\left|x_{n}-y_{n}\right| .
\end{aligned}
$$

Because, for every $i>n, x_{i+1}=\frac{x_{i}}{4}$ and $y_{i+1}=\frac{y_{i}}{4}$. Thus, we have that $\left|x_{i+1}-y_{i+1}\right|<\left|x_{i}-y_{i}\right|$ and, by c), $d\left(g_{1}(x), g_{1}(y)\right)<\varepsilon$.

Therefore, $g_{1}$ is continuous at every point of $R_{2}$.

3.3.3. Continuity of $g_{1}$ on $K_{2}$. Let $\varepsilon>0$ and $x=\left(x_{1}, x_{2}, \ldots\right) \in K_{2}$. Then there exists $N \in \mathbb{N}$ such that $\frac{1}{2^{N}}<\frac{\varepsilon}{2}$.

Since $x \in K_{2}, x_{n} \geq \frac{1}{2}$ for every $n \in \mathbb{N}$ and there exist $\delta_{0}, \delta_{1}$ and $\delta_{2}>0$ such that:

a) If $y \in K_{2}$ and $d(x, y)<\delta_{0}$, then $d\left(g_{1}(x), g_{1}(y)\right)<\varepsilon$; in fact, $d\left(g_{1}(x), g_{1}(y)\right)=0$.

b) If $s, t \in[0,1]$ and $|s-t|<\delta_{1}$, then $\left|f_{2}(s)-f_{2}(t)\right|<\frac{\varepsilon}{4}$.

c) If $y \in R_{2}$ and $d(x, y)<\delta_{2}$ then $\left|\pi_{N}(x)-\pi_{N}(y)\right|<\min \left\{\delta_{1}, \frac{1}{16}\right\}$.

Since $x_{n} \geq \frac{1}{2}$ for every $n \in \mathbb{N}$ : If $z=\left(z_{1}, z_{2}, \ldots, z_{k-1}, z_{k}, \ldots\right) \in \alpha_{k}^{2} \backslash \alpha_{k-1}^{2}$, then $z_{k}<\frac{1}{2}, z_{i}<\frac{1}{8}$ if $i \geq k+1$ and $z_{k-1} \geq \frac{1}{2}$; hence, $d(x, z)=\sum_{i=1}^{\infty} \frac{\left|x_{i}-z_{i}\right|}{2^{i}}=$ $\sum_{i=1}^{k} \frac{\left|x_{i}-z_{i}\right|}{2^{i}}+\sum_{i=k+1}^{\infty} \frac{\left|x_{i}-z_{i}\right|}{2^{i}}+\frac{x_{k+1}-z_{k+1}}{2^{k+1}} \geq \frac{x_{k+1}-z_{k+1}}{2^{k+1}}>\frac{3 / 8}{2^{k+1}}$; i.e.:

$$
\text { if } z \in \alpha_{k}^{2} \backslash \alpha_{k-1}^{2}, d(x, z)>\frac{3 / 8}{2^{k+1}} .
$$


Let $y \in X_{2}$ such that $d(x, y)<\delta=\min \left\{\delta_{0}, \delta_{2}, \frac{\frac{3}{8}}{2^{N+2}}\right\}$. By the choice of $\delta$ and by $(*), y \notin \cup_{k=1}^{N+1} \alpha_{k}^{2} \backslash \alpha_{k-1}^{2}$.

We consider two cases:

CASE 1. If $y \in K_{2}$.

This case is clear, since $g_{1}$ is constant on $K_{2}$.

CASE 2. If $y \notin K_{2}$.

Then $y \in \alpha_{j}^{2} \backslash \alpha_{j-1}^{2}$ for some $j>N+1$. Thus, $y_{j}<\frac{1}{2}$ and $y_{j-1} \geq \frac{1}{2}$. By c) and b), $\left|f_{2}\left(x_{N}\right)-f_{2}\left(y_{N}\right)\right|<\frac{\varepsilon}{2}$. Now, since $f_{1}\left(y_{j-1}\right)=\frac{1}{2}$,

$$
g_{1}(y)=\left(f_{1}^{j-2}\left(y_{j-1}\right), \ldots, f_{1}\left(y_{j-1}\right), y_{j-1}, \ldots\right)=\left(\frac{1}{2}, \ldots, \frac{1}{2}, y_{j-1}, \ldots\right) .
$$

This implies that

$$
\begin{aligned}
d\left(g_{1}(x), g_{1}(y)\right) & =d\left(\left(\frac{1}{2}, \frac{1}{2}, \ldots\right),\left(\frac{1}{2}, \ldots, \frac{1}{2}, y_{j-1}, \ldots\right)\right) \\
& =\sum_{i=1}^{j-2} \frac{\left|\frac{1}{2}-\frac{1}{2}\right|}{2^{i}}+\sum_{i=j-1}^{\infty} \frac{\left|\frac{1}{2}-y_{i}\right|}{2^{i}}<\frac{1}{2^{j}}<\frac{1}{2^{N}}<\varepsilon,
\end{aligned}
$$

by the choice of $N$. Thus, $d\left(g_{1}(x), g_{1}(y)\right)<\varepsilon$, and we obtain the continuity of $g_{1}$ at every point of $X_{2}$.

3.3.4. The map $g_{r}$. We define the function $g_{2}: X_{3} \rightarrow X_{2}$, for $x=$ $\left(x_{1}, x_{2}, x_{3}, \ldots\right) \in X_{3}$, by

$g_{2}(x)= \begin{cases}x, & \text { if } x \in \alpha_{2}^{3} ; \\ \left(y_{1}, y_{2}, \ldots, y_{n-2}, x_{n-1}, x_{n}, x_{n+1}, \ldots\right), & \text { if } x \in \alpha_{n}^{3} \backslash \alpha_{n-1}^{3}, n>2 ; \\ \bar{g}^{-1}\left(g_{1}(\bar{g}(x))\right), & \text { if } x \in K_{3} ;\end{cases}$

where $y_{i}=f_{1}^{n-1-i}\left(x_{n-1}\right)$.

In general, we define $g_{r}: X_{r+1} \rightarrow X_{r}$, for $x=\left(x_{1}, x_{2}, x_{3}, \ldots\right) \in X_{r+1}$, by $g_{r}(x)= \begin{cases}x, & \text { if } x \in \alpha_{2}^{r+1} \\ \left(y_{1}, y_{2}, \ldots, y_{n-2}, x_{n-1}, x_{n}, x_{n+1}, \ldots\right), & \text { if } x \in \alpha_{n}^{r+1} \backslash \alpha_{n-1}^{r+1}, n>2 \\ \bar{g}^{-1}\left(g_{r-1}(\bar{g}(x))\right), & \text { if } x \in K_{r+1}\end{cases}$ where $y_{i}=f_{1}^{n-1-i}\left(x_{n-1}\right)$.

3.3.5. The continuity of $g_{r}$. We only prove the continuity of $g_{2}$ since the continuity of $g_{r}$ is similar, except for the complexity of the cases and the indexes.

We omit the proof of the continuity of $g_{2}$ on the ray $R_{3}$, because it is similar to the continuity of $g_{1}$ on the ray $R_{2}$. We will prove de continuity of $g_{2}$ on $K_{3}$.

Let $x=\left(x_{1}, x_{2}, \ldots\right) \in K_{3}$. Then $x_{i} \geq \frac{1}{2}$ for every $i \in \mathbb{N}$. Since $K_{3}$ is homeomorphic to $X_{2}$, we have that $K_{3} \stackrel{2}{=} R_{1}^{3} \cup K_{3,2}$; i.e., $K_{3}$ is a 
compactification of a ray $R_{1}^{3}$ with remainder $K_{3,2}$, also $R_{1}^{3}=\cup_{n=1}^{\infty} \alpha_{n}^{3,2}$, where $\alpha_{n}^{3,2}=\left\{y \in K_{3}: y_{n}<\frac{3}{4}\right\}$.

Hence, we have that either $x \in \alpha_{s}^{3,2} \backslash \alpha_{s-1}^{3,2}$ for some $s \in \mathbb{N}$ or $x \in K_{3,2}$.

CASE 1. Suppose that $x \in \alpha_{s}^{3,2} \backslash \alpha_{s-1}^{3,2}$ for some $s \in \mathbb{N}$.

We note that

$$
\begin{aligned}
g_{2}(x) & =g_{2}\left(x_{1}, x_{2}, \ldots\right)=\bar{g}^{-1}\left[g_{1}\left(\bar{g}\left(x_{1}, x_{2}, \ldots\right)\right)\right] \\
& =\bar{g}^{-1}\left[g_{1}\left(g\left(x_{1}\right), g\left(x_{2}\right), \ldots, g\left(x_{s-2}\right), g\left(x_{s-1}\right), g\left(x_{s}\right), \ldots\right)\right] \\
& =\bar{g}^{-1}\left[f_{1}^{s-2}\left(g\left(x_{s-1}\right)\right), \ldots, f_{1}\left(g\left(x_{s-1}\right)\right), g\left(x_{s-1}\right), g\left(x_{s}\right), \ldots\right] \\
& =\left(g^{-1}\left(f_{1}^{s-2}\left(g\left(x_{s-1}\right)\right)\right), \ldots, g^{-1}\left(f_{1}\left(g\left(x_{s-1}\right)\right)\right), g^{-1}\left(g\left(x_{s-1}\right)\right),\right. \\
& \left.g^{-1}\left(g\left(x_{s}\right)\right), \ldots\right) .
\end{aligned}
$$

Also, we note that, since $x \in \alpha_{s}^{3,2} \backslash \alpha_{s-1}^{3,2}$, then $x_{i} \geq \frac{3}{4}$ for every $i \leq s-1$; and thus, $g\left(x_{i}\right) \geq \frac{1}{2}$ for every $i \leq s-1, f_{1}\left(g\left(x_{i}\right)\right)=\frac{1}{2}$ and $g^{-1}\left(f_{1}\left(g\left(x_{i}\right)\right)\right)=\frac{3}{4}$. $g_{2}(x)=\left(\frac{3}{4}, \ldots, \frac{3}{4}, x_{s-1}, x_{s}, \ldots\right)=\left(f_{2}^{s-1}\left(x_{s}\right), \ldots, f_{2}^{2}\left(x_{s}\right), f_{2}\left(x_{s}\right), x_{s}, \ldots\right)$. This follows from the definition of $f_{2}$.

Let $\varepsilon>0$. Then there exist positive numbers $\delta_{0}, \delta_{1}$ and $\delta_{2}$, every one less than $\frac{\varepsilon}{2}$, such that:

a) If $y \in K_{3}$ and $d(x, y)<\delta_{0}$, then $d\left(g_{2}(x), g_{2}(y)\right)<\varepsilon$.

b) If $r, t \in[0,1]$ and $|r-t|<\delta_{1}$, then $\sum_{i=1}^{s-1} \frac{\left|f_{2}^{i}(r)-f_{2}^{i}(t)\right|}{2^{i}}<\frac{\varepsilon}{2}$.

c) If $y \in R_{3}$ and $d(x, y)<\delta_{2}$, then $\left|\pi_{s}(x)-\pi_{s}(y)\right|<\min \left\{\delta_{1}, \frac{1}{64}\right\}$.

Let us note that, as in $(*)$ of the Section 3.3.3,

$$
\text { if } z \in \alpha_{k}^{3}, d(x, z)>\frac{\frac{3}{32}}{2^{k-1}} \text {. }
$$

Let $\delta=\min \left\{\delta_{0}, \delta_{2}, \frac{\frac{3}{32}}{2^{N+2}}\right\}$. Let $y \notin K_{3}$ such that $d(x, y)<\delta$. Then, by the choice of $\delta, y \in \alpha_{j}^{3} \backslash \alpha_{j-1}^{3}$ for some $j>s$.

Now, since $x_{s-1} \geq \frac{3}{4}$ and $\frac{9}{16}<x_{s}<\frac{3}{4}$, we obtain the following: $\left.f_{2}\right|_{\left[\frac{1}{2}, \frac{3}{4}\right]}=$ $\left.f_{3}\right|_{\left[\frac{1}{2}, \frac{3}{4}\right]}, \frac{1}{2}+\frac{1}{64}<x_{s+1}<\frac{9}{16}$ and $\frac{1}{2}+\frac{1}{4^{i+2}}<x_{i}<\frac{1}{2}+\frac{1}{4^{i+1}}$ where $i>s$.

By c) $\left|\pi_{s}(x)-\pi_{s}(y)\right|<\delta_{1}$; thus, $\frac{1}{2}+\frac{1}{64}<y_{s}<\frac{3}{4}$ and, by definition of $f_{3}$, $\frac{1}{2}+\frac{1}{4^{j+2}}<y_{j-1}<\frac{1}{2}+\frac{1}{4^{j}}$. So $y_{i} \in\left(\frac{1}{2}, \frac{5}{8}\right]$ where $i \in\{s, s+1, \ldots, j-1\}$ and $f_{2}\left(y_{i}\right) \in\left(\frac{1}{2}, \frac{3}{4}\right]$. Therefore, $y_{i+1}=f_{2}\left(y_{i}\right), i \in\{s, s+1, \ldots, j-1\}$. Now, if we calculate $g_{2}(y)=\left(f_{2}^{j-2}\left(y_{j-1}\right), \ldots, f_{2}^{j-1-s}\left(y_{j-1}\right), \ldots, f_{2}\left(y_{j-1}\right), y_{j-1}, \ldots\right)$, where the $s$-th coordinate is $f_{2}^{j-1-s}\left(y_{j-1}\right)$ then

$$
\begin{aligned}
g_{2}(y) & =\left(f_{2}^{j-2}\left(y_{j-1}\right), \ldots, f_{2}^{j-2-s}\left(y_{j-1}\right), y_{s}, y_{s+1}, \ldots, y_{j-1}, y_{j}, \ldots\right) \\
& =\left(f_{2}^{s-1}\left(y_{s}\right), \ldots, f_{2}\left(y_{s}\right), y_{s}, \ldots\right) .
\end{aligned}
$$


Thus,

$$
\begin{aligned}
d\left(g_{2}(x),\right. & \left.g_{2}(y)\right) \\
= & d\left(\left(f_{2}^{s-1}\left(x_{s}\right), \ldots, f_{2}^{2}\left(x_{s}\right), f_{2}\left(x_{s}\right), x_{s}, \ldots\right),\right. \\
& \left.\left(f_{2}^{s-1}\left(y_{s}\right), \ldots, f_{2}\left(y_{s}\right), y_{s}, \ldots\right)\right) \\
= & \sum_{i=1}^{s-1} \frac{\left|f_{2}^{i}\left(x_{s}\right)-f_{2}^{i}\left(y_{s}\right)\right|}{2^{i}}+\sum_{i=s}^{\infty} \frac{\left|x_{s}-y_{s}\right|}{2^{i}}<\frac{\varepsilon}{2}+\delta_{1}<\varepsilon .
\end{aligned}
$$

By b), the first sum is less than $\frac{\varepsilon}{2}$. Regarding second sum, we note that $\left.f_{3}\right|_{\left[\frac{1}{2}, \frac{5}{8}\right]}$ is the monotone map $f_{3}(t)=4 t-\frac{3}{2}$; and thus, if $i>s,\left|x_{i+1}-y_{i+1}\right|=$ $\left|\frac{x_{i}}{4}+\frac{3}{8}-\frac{y_{i}}{4}-\frac{3}{8}\right|=\left|\frac{x_{i}}{4}-\frac{y_{i}}{4}\right|<\left|x_{i}-y_{i}\right|$. Then $\left|x_{s}-y_{s}\right|>\left|x_{i}-y_{i}\right|$ for every $i>s$, thus, the second sum is less than $\left|x_{s}-y_{s}\right|$ and, by c) it is less than $\delta_{1}$. Therefore, $g_{2}$ is continuous at $x$.

CASE 2. If $x \in K_{3}$.

In this case

$$
\begin{aligned}
g_{2}(x) & =g_{2}\left(x_{1}, x_{2}, \ldots\right)=\bar{g}^{-1}\left[g_{1}\left(\bar{g}\left(x_{1}, x_{2}, \ldots\right)\right)\right] \\
& =\bar{g}^{-1}\left[g_{1}\left(g\left(x_{1}\right), g\left(x_{2}\right), \ldots, g\left(x_{s-2}\right), g\left(x_{s-1}\right), g\left(x_{s}\right), \ldots\right)\right]
\end{aligned}
$$

and, since $x_{i} \geq \frac{3}{4}$ for every $i, g\left(x_{i}\right) \geq \frac{1}{2}$. Then $g_{2}(x)=\bar{g}^{-1}\left(\frac{1}{2}, \frac{1}{2}, \ldots\right)=$ $\left(g^{-1}\left(\frac{1}{2}\right), g^{-1}\left(\frac{1}{2}\right), \ldots\right)=\left(\frac{3}{4}, \frac{3}{4}, \ldots\right)$.

Let $N \in \mathbb{N}$ such that $\frac{1}{2^{N}}<\frac{\varepsilon}{2}$. By $(* *)$, if $z \in \alpha_{k}^{3}$ then $d(x, z)>\frac{\frac{3}{32}}{2^{k-1}}$. Then, let $\delta<\frac{\frac{3}{32}}{2^{N-1}}$, thus, if $y \in R_{3}$ and $d(x, y)<\delta$, then $y \in \alpha_{j}^{3} \backslash \alpha_{j-1}^{3}$, where $j>N+1$; and thus, $g_{2}(y)=\left(f_{2}^{j-2}\left(y_{j-1}\right), \ldots, f_{2}\left(y_{j-1}\right), y_{j-1}, y_{j}, \ldots\right)$. We note that $y_{j-1} \geq \frac{1}{2}$ and $y_{i} \geq \frac{1}{2}$ for every $i \leq j-1$. By c) $\left|y_{N}-x_{N}\right|<\delta_{1}<\frac{1}{32}$, but $x_{N}=\frac{3}{4}$. Thus, $y_{N}>\frac{5}{8}$. Hence, $y_{N-1} \geq \frac{3}{4}$ and $y_{i} \geq \frac{3}{4}$ for every $i<N$.

Since $y_{i} \geq \frac{1}{2}$ for every $i \leq j-1$, we have two subcases:

CASE 2.1. If for some $i \in\{N, \ldots, j-1\}, y_{i} \geq \frac{3}{4}$.

In this case $g_{2}(y)=\left(\frac{3}{4}, \frac{3}{4}, \ldots, \frac{3}{4}, y_{i}, y_{i+1}, \ldots, y_{j-1}, y_{j}, \ldots\right)$.

CASE 2.2. The first coordinate $i$ where $y_{i} \geq \frac{3}{4}$ is $i=N-1$.

In both cases, the image of $y$ is $\frac{3}{4}$ in the first $(N-1)$ coordinates. Therefore,

$$
\begin{aligned}
d\left(g_{2}(x), g_{2}(y)\right) & =\left(\left(\frac{3}{4}, \frac{3}{4}, \ldots\right)\left(\frac{3}{4}, \ldots, \frac{3}{4}, y_{i}, y_{i+1}, \ldots\right)\right) \\
& =\sum_{i=1}^{N-1} \frac{\left|\frac{3}{4}-\frac{3}{4}\right|}{2^{i}}+\sum_{i=N}^{\infty} \frac{\left|\frac{3}{4}-\pi_{i}\left(g_{2}(y)\right)\right|}{2^{i}}<\varepsilon
\end{aligned}
$$

Since the first sum is zero and the other is less than $\frac{1}{2^{N}}$, then $d\left(g_{2}(x), g_{2}(y)\right)<$ $\varepsilon$ and we obtain the continuity of $g_{2}$ at every point of $X_{3}$. 
3.3.6. The function $g_{r}$ is monotone. We start proving that $\left.g_{r}\right|_{R_{r+1}}$ is a homeomorphism. Then we show that $\left.g_{r}\right|_{R_{s}^{r+1}}$ is a homeomorphism. Finally we will prove that $g_{r}$ is monotone. Recall

$g_{r}(x)= \begin{cases}x, & \text { if } x \in \alpha_{2}^{r+1} ; \\ \left(y_{1}, y_{2}, \ldots, y_{n-2}, x_{n-1}, x_{n}, x_{n+1}, \ldots\right), & \text { if } x \in \alpha_{n}^{r+1} \backslash \alpha_{n-1}^{r+1}, n>2 ; \\ \bar{g}^{-1}\left(g_{r-1}(\bar{g}(x))\right), & \text { if } x \in K_{r+1} ;\end{cases}$

where $y_{i}=f_{1}^{n-1-i}\left(x_{n-1}\right)$.

Proposition 3.1. The image of $R_{r+1}$ under the function $g_{r}$ is $R_{r}$.

Proof. Let $x=\left(x_{1}, x_{2}, \ldots\right) \in R_{r+1}$. Then either $x \in \alpha_{2}^{r+1}$ or $x \in$ $\alpha_{n}^{r+1} \backslash \alpha_{n-1}^{r+1}$ for some $n>2$. If $x \in \alpha_{2}^{r+1}$, then $g_{r}(x)=x$ and, since $x_{2}<\frac{1}{2}, g_{2}(x) \in \alpha_{2}^{r} \subset R_{r}$. On the other hand, if $x \in \alpha_{n}^{r+1} \backslash \alpha_{n-1}^{r+1}$, we obtain that $g_{r}(x)=\left(f_{r}^{n-2}\left(x_{n-1}\right), f_{r}^{n-1}\left(x_{n-1}\right), \ldots, f_{r}\left(x_{n-1}\right), x_{n-1}, x_{n}, \ldots\right)$, which is a point of $X_{r}$ such that its $n$-th coordinate, $x_{n}$, is less than $\frac{1}{2}$, and every coordinate $i<n$, satisfies that $x_{i} \geq \frac{1}{2}$. Thus, $g_{r}(x) \in \alpha_{n}^{r} \backslash \alpha_{n-1}^{r}$; i.e., the image of $x$ under $g_{r}$ is in $R_{r}$.

If $y=\left(y_{1}, y_{2}, \ldots\right) \in R_{r}$, then either $y \in \alpha_{2}^{r}$ or $y \in \alpha_{n}^{r} \backslash \alpha_{n-1}^{r}$ for some $n>2$. If $y \in \alpha_{2}^{r}$ then, if we take $x=y$, we obtain that $g_{r}(x)=y$, if $y \in \alpha_{n}^{r} \backslash \alpha_{n-1}^{r}$, then, if we take the point $x=$ $\left(f_{r+1}^{n-2}\left(y_{n-1}\right), f_{r+1}^{n-1}\left(y_{n-1}\right), \ldots, f_{r+1}\left(y_{n-1}\right), y_{n-1}, y_{n}, \ldots\right)$, we obtain that $x$ is a point in $\alpha_{n}^{r+1} \backslash \alpha_{n-1}^{r+1}$, which satisfies the following

$$
g_{r}(x)=\left(f_{r}^{n-2}\left(y_{n-1}\right), f_{r}^{n-1}\left(y_{n-1}\right), \ldots, f_{r}\left(y_{n-1}\right), y_{n-1}, y_{n}, \ldots\right)=y .
$$

This shows that every point of $R_{r}$ is the image of a point of $R_{r+1}$.

Proposition 3.2. $\left.g_{r}\right|_{R_{r+1}}$ is injective.

Proof. Let $x, y \in R_{r+1}$ and suppose that $g_{r}(x)=g_{r}(y)$. By definition of the map $g_{r}$, it is clear that, either $x, y \in \alpha_{2}^{r+1}$ or there is an $n>2$, such that, $x, y \in \alpha_{n}^{r+1} \backslash \alpha_{n-1}^{r+1}$.

In the first case: $x=g_{r}(x)=g_{r}(y)=y$. In the second case:

$$
g_{r}(x)=\left(f_{r}^{n-2}\left(x_{n-1}\right), f_{r}^{n-1}\left(x_{n-1}\right), \ldots, f_{r}\left(x_{n-1}\right), x_{n-1}, x_{n}, \ldots\right)
$$

and

$$
g_{r}(y)=\left(f_{r}^{n-2}\left(y_{n-1}\right), f_{r}^{n-1}\left(y_{n-1}\right), \ldots, f_{r}\left(y_{n-1}\right), y_{n-1}, y_{n}, \ldots\right),
$$

the second equality is true only when $x=y$.

THEOREM 3.3. $\left.g_{r}\right|_{R_{r+1}}$ is a homeomorphism.

Proof. By the Propositions 3.1 and 3.2, $\left.g_{r}\right|_{R_{r+1}}$ is a bijective function. The proof that $\left.g_{r}\right|_{R_{r+1}}$ is continuous is similar to the continuity of $\left.g_{1}\right|_{R_{2}}$ (which is in the Section 3.3.2). 
Moreover, the inverse function of $\left.g_{r}\right|_{R_{r+1}}$ is continuous, which is defined by

$g_{r}^{-1}(x)= \begin{cases}x, & \text { if } x \in \alpha_{2}^{r} ; \\ \left(f_{r+1}^{n-2}\left(x_{n-1}\right), \ldots, f_{r+1}\left(x_{n-1}\right), x_{n-1}, x_{n}, \ldots\right) & \text { if } x \in \alpha_{n}^{r} \backslash \alpha_{n-1}^{r} ;\end{cases}$

where $x=\left(x_{1}, x_{2}, \ldots\right) \in R_{r}$. The proof of the continuity is similar to proof of the continuity of $g_{r}$.

Now, we will show that $\left.g_{2}\right|_{R_{1}^{3}}$ is a homeomorphism from $R_{1}^{3}$ onto $R_{1}^{2}$.

First, we note that $X_{r}=\overline{R_{r}}=R_{r} \cup K_{r}$ and $K_{r}$ is homeomorphic to $X_{r-1}$. Since $X_{1}=R_{1} \cup K_{1}, X_{2}=R_{2} \cup R_{1}^{2} \cup K_{1}^{2}, X_{3}=R_{3} \cup R_{1}^{3} \cup R_{2}^{3} \cup K_{1}^{3}$ and, in general $X_{r}=R_{r} \cup R_{1}^{r} \cup R_{2}^{r} \cup \cdots \cup R_{r-1}^{r} \cup K_{1}^{r}$. We note that $R_{r-1}^{r} \cup K_{1}^{r}$ is homeomorphic to $X_{1}$ and, in general, that $R_{r-s}^{r} \cup \cdots \cup R_{r-1}^{r} \cup K_{1}^{r}$ is homeomorphic to $X_{s}$.

Since $K_{3}$ is homeomorphic to $X_{2}$, then $K_{3}=R_{1}^{3} \cup K_{3,2}$ and $K_{3,2}$ is homeomorphic to $X_{1}$.

Recall the definition of $g_{2}$, if $x=\left(x_{1}, x_{2}, \ldots\right) \in X_{3}$, then

$g_{2}(x)= \begin{cases}x, & \text { if } x \in \alpha_{2}^{3} ; \\ \left(y_{1}, y_{2}, \ldots, y_{n-2}, x_{n-1}, x_{n}, x_{n+1}, \ldots\right), & \text { if } x \in \alpha_{n}^{3} \backslash \alpha_{n-1}^{3}, n>2 ; \\ \bar{g}^{-1}\left(g_{1}(\bar{g}(x))\right), & \text { if } x \in K_{3} ;\end{cases}$

where $y_{i}=f_{1}^{n-1-i}\left(x_{n-1}\right)$.

Let us see the image of one element in $R_{1}^{3}$ under $g_{2}$.

If $x \in K_{3}$, then either $x \in \alpha_{s}^{3,2} \backslash \alpha_{s-1}^{3,2}$ for some $s \in \mathbb{N}$ or $x \in K_{3,2}$.

If $x \in \alpha_{s}^{3,2} \backslash \alpha_{s-1}^{3,2}$ for some $s \in \mathbb{N}$, then

$$
\begin{aligned}
g_{2}(x) & =\bar{g}^{-1}\left(g_{1}(\bar{g}(x))\right) \\
& =\left(g^{-1}\left(f_{1}^{s-2}\left(g\left(x_{s-1}\right)\right)\right), \ldots, g^{-1}\left(f_{1}\left(g\left(x_{s-1}\right)\right)\right), x_{s-1}, x_{s}, \ldots\right) .
\end{aligned}
$$

But $\frac{1}{2} \leq x_{s} \leq \frac{3}{4}$ and $x_{s-1} \geq \frac{3}{4}$. In fact, $x_{i} \geq \frac{3}{4}$ for every $i<s$, and $\frac{1}{2} \leq x_{i} \leq \frac{3}{4}$ for every $i \geq s$. Then $g_{2}(x)=\bar{g}^{-1}\left(g_{1}(\bar{g}(x))\right)=$ $\bar{g}^{-1}\left(g_{1}\left(g\left(x_{1}\right), g\left(x_{2}\right), \ldots, g\left(x_{s-1}\right), g\left(x_{s}\right), \ldots\right)\right)$.

Since $0 \leq g\left(x_{s}\right) \leq \frac{1}{2}, g\left(x_{s-1}\right) \geq \frac{1}{2}$; in fact, $0 \leq g\left(x_{i}\right)<\frac{1}{2}$ if $i \geq s$ and $g\left(x_{i}\right) \geq \frac{1}{2}$ if $i<s$. Then $\bar{g}(x) \in \alpha_{s}^{2} \backslash \alpha_{s-1}^{2}$. Hence,

$$
\begin{aligned}
g_{2}(x) & =\bar{g}^{-1}\left(f_{1}^{s-2}\left(g\left(x_{s-1}\right)\right), \ldots, f_{1}\left(g\left(x_{s-1}\right)\right), g\left(x_{s-1}\right), g\left(x_{s}\right), \ldots\right) \\
& =\left(g^{-1}\left(f_{1}^{s-2}\left(g\left(x_{s-1}\right)\right)\right), \ldots, g^{-1}\left(f_{1}\left(g\left(x_{s-1}\right)\right)\right), x_{s-1}, x_{s}, \ldots\right) .
\end{aligned}
$$

Since $x_{s-1} \geq \frac{1}{2}$ and $x_{i} \geq \frac{1}{2}$ for every $i$, and, since $x_{s-1} \geq \frac{3}{4}$, then $f_{2}\left(x_{s-1}\right)=$ $\frac{3}{4}$. We note that $g^{-1}\left(f_{1}^{2}\left(g\left(x_{s-1}\right)\right)\right)=g^{-1}\left(f_{1}\left(g\left(g^{-1}\left(f_{1}\left(g\left(x_{s-1}\right)\right)\right)\right)\right)\right)=$ $g^{-1}\left(f_{1}\left(g\left(f_{2}\left(x_{s-1}\right)\right)\right)\right)$.

Moreover, $g^{-1}\left(f_{1}^{n}\left(g\left(x_{s-1}\right)\right)\right)=f_{2}^{n}\left(x_{s-1}\right)$. Thus,

$$
g_{2}(x)=\left(f_{2}^{s-1}\left(x_{s-1}\right), \ldots, f_{2}\left(x_{s-1}\right), x_{s-1}, x_{s}, \ldots\right) .
$$


Since $x_{s-1} \geq \frac{3}{4}$, it follows, from the definition of $f_{2}$, that

$$
g_{2}(x)=\left(\frac{3}{4}, \frac{3}{4}, \ldots, \frac{3}{4}, x_{s-1}, x_{s}, \ldots\right) .
$$

This implies $g_{2}(x) \in R_{1}^{2}$.

Moreover, if $y=\left(y_{1}, y_{2}, \ldots\right) \in R_{1}^{2}$, then $y_{i} \geq \frac{1}{2}$ for every $i \in \mathbb{N}$ and $y_{s} \leq \frac{3}{4}$ for some $s \in \mathbb{N}$; i.e., $y=\left(f_{2}^{s-1}\left(y_{s-1}\right), \ldots, f_{3}\left(y_{s-1}\right), y_{s-1}, \ldots\right)$; hence, if we define $x=\left(f_{3}^{s-3}\left(y_{s-1}\right), \ldots, f_{3}\left(y_{s-1}\right), y_{s-1}, y_{s}, \ldots\right)$, then $\frac{1}{2} \leq y_{s}<\frac{3}{4}$ and $y_{s-1} \geq \frac{3}{4}$; thus, $g_{2}(x)=y$.

Now we will prove that $\left.g_{2}\right|_{R_{1}^{3}}$ is injective.

Let $x, y \in R_{1}^{3}$ and let $g_{2}(x)=g_{2}(y)$. By $(\#)$, we obtain that $\left(\frac{3}{4}, \frac{3}{4}, \ldots, \frac{3}{4}, x_{s-1}, x_{s}, \ldots\right)=\left(\frac{3}{4}, \frac{3}{4}, \ldots, \frac{3}{4}, y_{s-1}, y_{s}, \ldots\right)$, where $x_{i}=y_{i}$ for every $i \geq s-1$, but $x_{i}=f_{3}^{s-1-i}\left(x_{s-1}\right)$ and $y_{i}=f_{3}^{s-1-i}\left(y_{s-1}\right)$ for every $i<s-1$. Hence, $x=y$.

Thus, $\left.g_{2}\right|_{R_{1}^{3}} ^{-1}$ defined for $x=\left(x_{1}, x_{2}, \ldots\right) \in R_{1}^{2}$ by

$$
\left.g_{2}\right|_{R_{2}^{3}} ^{-1}(x)=\left(f_{3}^{s-1}\left(x_{s-1}\right), \ldots, f_{3}\left(x_{s-1}\right), x_{s-1}, x_{s}, \ldots\right),
$$

is continuous. The proof of the continuity is similar to the proof of the continuity of $\left.g_{2}\right|_{R_{2}}$.

The following are Corollaries to Theorem 3.3.

COROLlary 3.4. $\left.g_{2}\right|_{R_{1}^{3}}$ is a homeomorphism.

Corollary 3.5. $\left.g_{r}\right|_{R_{s}^{r}}, s<r$ is a homeomorphism.

Now, we will show, by induction, that $g_{r}$ is monotone.

Proposition 3.6. $g_{r}$ is monotone.

Proof. We will prove that $g_{1}$ is monotone. Let $x=\left(x_{1}, x_{2}, \ldots\right) \in X_{1}$. If $x \in R_{1}$, by Proposition 3.1, $g_{1}^{-1}(x) \in R_{2}$ and, by Proposition $3.2, g_{1}^{-1}(x)$ is a point; and thus, connected. Now, if $x \in K_{1}$; i.e., $x=\left(\frac{1}{2}, \frac{1}{2}, \ldots\right), g_{1}^{-1}(x)=K_{2}$ (it is a consequence of Proposition 3.1), which is connected. Thus, $g_{1}$ is monotone.

Now, if $r>1$, let $x=\left(x_{1}, x_{2}, \ldots\right) \in X_{r}$. If $x \in R_{r}$, by Proposition $3.1, g_{r}^{-1}(x) \in R_{r+1}$ and, by Proposition 3.2, $g_{r}^{-1}(x)$ is a point; and thus, it is connected. If $x \in K_{r}$, then, as a consequence of Proposition 3.1, $g_{r}^{-1}(x) \subseteq K_{r+1}$, from where we obtain that $g_{r}^{-1}(x)=\left(\left.g_{r}\right|_{K_{r+1}}\right)^{-1}(x)$. But $\left.g_{r}\right|_{K_{r+1}}=\bar{g}^{-1} \circ g_{r-1} \circ \bar{g}$, which is a composition of monotone maps, so $g_{r}^{-1}(x)$ is connected. Thus, $g_{r}$ is monotone.

3.4. The continuum. Let $X=\lim \left\{X_{n}, g_{n}\right\}$, where $X_{n}$ is the $n$-th factor space that we defined before and $g_{n}$ is the bonding map between the spaces $X_{n+1}$ and $X_{n}$.

First, we will prove that $X$ is a compactification of a ray. 
Let $R=\lim \left\{R_{n},\left.g_{n}\right|_{R_{n+1}}\right\}$. We note that $R$ is well defined, because $\left.g_{n}\right|_{R_{n+1}}$ is a homeomorphism from $R_{n+1}$ onto $R_{n}$. On the other hand, if $x=\left(x_{1}, x_{2}, \ldots\right) \in R$, then $x_{i} \in R_{i}$ where $x_{i}=\left(x_{1}^{i}, x_{2}^{i}, \ldots, x_{s-1}^{i}, x_{s}^{i}, \ldots\right)$. Recall that $R_{i}=\alpha_{1}^{i} \cup \cup_{n=2}^{\infty}\left(\alpha_{n}^{i} \backslash \alpha_{n-1}^{i}\right)$, which implies that $x_{i} \in \alpha_{s}^{i} \backslash \alpha_{s-1}^{i}$ for some $s \in \mathbb{N}$.

Hence, $x_{i}=\left(f_{i}^{s-2}\left(x_{s-1}^{i}\right), \ldots, f_{i}\left(x_{s-1}^{i}\right), x_{s-1}^{i}, x_{s}^{i}, \ldots\right)$ and

$$
\begin{aligned}
& \left(f_{i}^{s-2}\left(x_{s-1}^{i}\right), \ldots, f_{i}\left(x_{s-1}^{i}\right), x_{s-1}^{i}, x_{s}^{i}, \ldots\right) \\
& \quad=\left(f_{1}^{s-2}\left(x_{s-1}^{1}\right), \ldots, f_{1}\left(x_{s-1}^{1}\right), x_{s-1}^{1}, x_{s}^{1}, \ldots\right) .
\end{aligned}
$$

We obtain the last equality because $\left.g_{n}\right|_{R_{n+1}}$ is a homeomorphism for every $n \in\{1,2, \ldots, i-1\}$. Then there is a homeomorphism from $R$ onto $R_{1}$, defined by the first projection map restricted to $R, \pi_{1}(x)=x_{1}$, because $\left.\pi_{1}\right|_{R}$ is bijective, continuous and its inverse, defined by $\pi_{1}^{-1}\left(x_{1}\right)=$ $\left(x_{1}, g_{1}^{-1}\left(x_{1}\right), g_{2}^{-1}\left(g_{1}^{-1}\left(x_{1}\right)\right), \ldots\right)$ is continuous, since it is continuous at each coordinate. Then we obtain that $R$ is a ray.

Now, if $x=\left(x_{1}, x_{2}, \ldots\right) \in X \backslash R$, then $x_{i} \notin R_{i}$ for some $i$, but, by Proposition 3.1, we obtain that $x_{j} \notin R_{j}$ for every $j \in \mathbb{N}$.

We will show that, given a positive number $\varepsilon$, there exists a point $y \in R$ such that $d(x, y)<\varepsilon$.

Let $\varepsilon>0$. Then there exists $N \in \mathbb{N}$ such that $\frac{1}{2^{N}}<\frac{\varepsilon}{2}$ and there exists $\delta>0$ such that, if $d(s, t)<\delta$ then

$$
\sum_{i=1}^{N-1} \frac{d\left(g_{N-i}\left(\ldots\left(g_{N-1}(s)\right)\right), g_{N-1}\left(\ldots,\left(g_{N-1}(t)\right)\right)\right)}{2^{i}}<\frac{\varepsilon}{2} .
$$

Let $y_{N} \in R_{N}$ such that $d\left(x_{N}, y_{N}\right)<\delta$ (it is possible, because $\overline{R_{N}}=X_{N}$ ).

Since $\left.g_{n}\right|_{R_{n+1}}$ is a homeomorphism from $R_{n+1}$ onto $R_{n}$, we define $y \in R$, where $y=\left(y_{1}, y_{2}, \ldots, y_{N}, \ldots\right)$ such that $y_{i}=g_{N-i}\left(\ldots\left(g_{N-1}\left(y_{N}\right)\right)\right)$ if $i<N$ and $y_{i}=g_{i}^{-1}\left(\ldots\left(g_{N}^{-1}\left(y_{N}\right)\right)\right)$ if $i>N$.

Then, we obtain a point $y \in R$ and

$$
\begin{aligned}
d(x, y)= & d\left(\left(x_{1}, x_{2}, \ldots\right),\left(y_{1}, y_{2}, \ldots\right)\right) \\
= & \sum_{i=1}^{N-1} \frac{d\left(g_{N-i}\left(\ldots\left(g_{N-1}\left(y_{N}\right)\right)\right), g_{N-1}\left(\ldots\left(g_{N-1}\left(x_{N}\right)\right)\right)\right)}{2^{i}} \\
& +\sum_{i=N}^{\infty} \frac{d\left(x_{i}, y_{i}\right)}{2^{i}}<\varepsilon .
\end{aligned}
$$

THEOREM 3.7. $X$ is 2-equivalent.

Proof. Let $q_{i}=\left(1-\frac{1}{2^{i}}, 1-\frac{1}{2^{i}}, \ldots\right)$ for every $i \in\{1,2, \ldots\}$ and $q=$ $\left(q_{1}, q_{2}, \ldots\right)$. By the definition of $X_{i}$ and $X, q_{i} \in X_{i}$ and $q \in X$. 
If $A$ is a subcontinuum of $X$, we will prove that, if $q \notin A$, then $A$ is an arc and if $q \in A$, then $A$ is homeomorphic to $X$. Let $A$ a nondegenerate subcontinuum of $X$.

CASE 1. $q \notin A$.

Then $q_{i} \notin A_{i}=\pi_{i}(A)$, for some $i$, which implies that $A_{i}$ is a subcontinuum of $X_{i}$, such that $X_{i}$ does not contain $K_{i}$ and since $X_{i}=$ $R_{i} \cup R_{i-1}^{i} \cup \ldots \cup R_{1}^{i} \cup\left\{q_{i}\right\}$, we obtain that either $A_{i} \subseteq R_{i}$ or $A_{i} \subseteq R_{s}^{i}$ for some $s \in\{1,2, \ldots, i-1\}$. From here we obtain that $A_{i}$ is an arc contained in a ray; thus, $A_{i+1}=\pi_{i+1}(A)$ is contained in either $R_{i+1}$ or $A_{i+1} \subseteq R_{s}^{i+1}$ for some $s \in\{1,2, \ldots, i-1\}$ such that $A_{i} \subseteq R_{s}^{i}$ (because, with the bonding map the image of $R_{i+1}$ is $R_{i}$ and the image of every $R_{s}^{i+1}$ is $R_{s}$ ).

In general if $j>i$, either $\pi_{j}(A)=A_{j} \subseteq R_{j}$ or $A_{j} \subseteq R_{s}^{j}$ for the $s \in$ $\{1,2, \ldots, i-1\}$ which satisfies that $A_{i} \subseteq R_{s}^{i}$. Therefore, $A$ is an arc.

CAsE 2. $q \in A$.

Then $q_{i} \in A_{i}=\pi_{i}(A)$ for every $i \in \mathbb{N}$.

If $A_{1}$ is nondegenerate, $A_{1}$ is an arc, such that, one of its end points is $q_{1}$, then $A_{1}$ is homeomorphic to $X_{1}$.

Let $a_{1}$ be the other end point of $A_{1} . a_{1} \in R_{1}$ because $A_{1} \neq\left\{\left(\frac{1}{2}, \frac{1}{2}, \ldots\right)\right\}=$ $\left\{q_{1}\right\}$. Let $a_{2}=g_{1}^{-1}\left(a_{1}\right)$. Recall that $\left.g_{r}\right|_{R_{r+1}}$ is a homeomorphism, $a_{2} \in A_{2}$ and, since $q_{2} \in A_{2}$, we obtain that $A_{2} \cap R_{2} \neq \emptyset$ and $A_{2} \cap K_{2} \neq \emptyset$. Moreover, since $K_{2}$ is the remainder, $K_{2} \subseteq A$. Then $A_{2}$ is homeomorphic to $X_{2}$.

In general, $a_{i}=g_{i-1}^{-1}\left(\ldots\left(g_{1}^{-1}\left(a_{1}\right)\right)\right) \in A_{i}$ and, since $q_{i} \in A_{i}$, we obtain that $A_{i}$ is a compactification of a subray of $R_{i}$, i.e., $A_{i}$ is homeomorphic to $X_{i}$. Thus, $A$ is homeomorphic to $X$.

Now, let us suppose that $A_{1}$ is degenerate. Since $A$ is nondegenerate, let $t>1$ be the minimum of the numbers, such that $A_{t}$ is nondegenerate. Then $A_{t}$ is an arc contained in $K_{t}$ (recall that $A_{t-1}=\pi_{t-1}(A)=\left\{q_{t-1}\right\}$, where $a_{t}$ and $q_{t}$ are its end points). Then $a_{t} \in R_{t-1}^{t}$.

On the other hand, in $X_{t+1}, a_{t+1} \in R_{t-1}^{t+1}$, and $A_{t+1}$ does not intersect any ray neither $R_{t+1}$ nor $R_{j}^{t+1}$ for every $j<t-1$.

Since $A_{t+1}$ intersect to $K_{t+1}$, it is a compactification of a subray of $R_{t-1}^{t+1}$, thus $A_{t+1}$ is homeomorphic to $X_{1}$. If we continue with this process, we obtain that $A_{j}$ is homeomorphic to $X_{j-t}$ for every $j>t$, and thus $A$ is homeomorphic to $X$.

TheOREM 3.8. $X$ is a Kelley continuum.

Proof. Note that $X=\lim _{\longleftarrow}\left\{X_{n}, g_{n}\right\}$, each $X_{n}$ is a Kelley continuum and every bonding map $g_{n}$ is confluent. By the Theorem 1.3, $X$ is a Kelley continuum.

ACKNOWLEDGEMENTS.

The author thanks the referee for his/her suggestions. Thanks, too, to 
Rocío Leonel and Sergio Macías for their very useful help in the preparation of this paper.

\section{REFERENCES}

[1] R. A. Beane and W. J. Charatonik, Kelley remainders of $[0, \infty)$, Topology Proc. 32 (2008), 101-114.

[2] R. Bennett, On inverse limit sequences, Master's Thesis, University of Tennesse, 1962.

[3] J. J. Charatonik and W. J. Charatonik, Fans with the property of Kelley, Topology Appl. 29 (1988), 73-78.

[4] W. J. Charatonik, Inverse limits of smooth continua, Comment. Math. Univ. Carolin. 23 (1982), 183-191.

[5] H. Cook, Tree-likeness of hereditarily equivalent continua, Fund. Math. 68 (1970), 203-205.

[6] G. W. Henderson, Proof that every compact decomposable continuum which is topologically equivalent to each of its nondegenerate subcontinua is an arc, Ann. of Math. (2) $\mathbf{7 2}$ (1960), 421-428.

[7] W. T. Ingram, Periodicity and indecomposability, Proc. Amer. Math. Soc. 123 (1995), 1907-1916.

[8] W. T. Ingram, Families of inverse limits on [0, 1], Topology Proc. 27 (2003), 189-201.

[9] S. Macías, Topics on continua, Chapman \& Hall/CRC, Boca Raton, 2005.

[10] T. Mackowiak, Singular arc-like continua, Dissertationes Math. 257 (1986), 40 pp.

[11] W. S. Mahavier, Continua with only two topologically different subcontinua, Topology Appl. 94 (1999), 243-252.

[12] S. Mazurkiewicz, Problem 14, Fund. Math. 2 (1921), p. 286.

[13] E. E. Moise, An indecomposable plane continuum which is homeomorphic to each of its nondegenerate subcontinua, Trans. Amer. Math. Soc. 63 (1948), 581-594.

[14] S. B. Nadler Jr., Continuum theory. An introduction, Marcel Deker, Inc., New York, 1992.

[15] S. B. Nadler Jr., Hyperspaces of sets. A text with research questions. Aportaciones Matemáticas, Serie Textos 33, Sociedad Matemática Mexicana, Mex́ico, 2006.

[16] G. T. Whyburn, A continuum every subcontinuum of which separates the plane, Amer. J. Math. 52 (1930), 319-330.

C. Islas

Department of Mathematics

Faculty of Sciences

Universidad Autónoma de la Ciudad de México

04510 México DF

México

E-mail: islas@matem.unam.mx \& carlos.islas@uacm.edu.mx

Received: 5.5.2009.

Revised: 2.2.2010. \& 7.4.2010. 